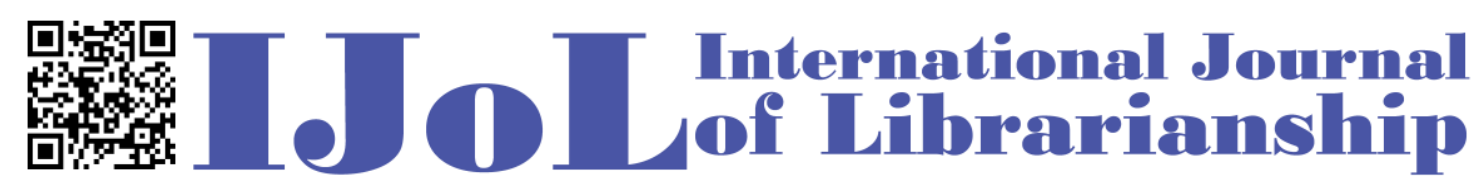

ISSN: 2474-3542 Journal homepage: http://journal.calaijol.org

\title{
Overview of Development and Recent Trends in Bibliometrics and Research Evaluation
}

\section{Yongming Wang}

\begin{abstract}
:
This paper is an overview of bibliometrics, a subfield of library and information science. It briefly explains what bibliometrics is and why it is important in research evaluation and impact analysis. It summarizes the latest development and trends over the past decade. Three major trends are identified and discussed. They are alternative metrics, responsible use of bibliometrics and responsible research evaluation movement, and application of artificial intelligence (AI) and machine learning in bibliometrics practice.
\end{abstract}

To cite this article:

Wang, Y. (2021). Overview of development and recent trends in bibliometrics and research evaluation. International Journal of Librarianship, 6(1), 105-108.

https://doi.org/10.23974/ijol.2021.vol6.1.195

To submit your article to this journal:

Go to https://ojs.calaijol.org/index.php/ijol/about/submissions 


\title{
Overview of Development and Recent Trends in Bibliometrics and Research Evaluation
}

\author{
Yongming Wang \\ The College of New Jersey (TCNJ), NJ, USA
}

\begin{abstract}
This paper is an overview of bibliometrics, a subfield of library and information science. It briefly explains what bibliometrics is and why it is important in research evaluation and impact analysis. It summarizes the latest development and trends over the past decade. Three major trends are identified and discussed. They are alternative metrics, responsible use of bibliometrics and responsible research evaluation movement, and application of artificial intelligence (AI) and machine learning in bibliometrics practice.
\end{abstract}

\section{HISTORICAL REVIEW}

Bibliometrics is the use of citation data to evaluate the importance and impact of scientific articles and other published works, including the authors of these articles and the journals where the works are published. Citation analysis is the most frequently used method in bibliometrics. Bibliometrics is sometimes also referred to as scientometrics or informetrics.

By measuring the contribution and impact of scientists and researchers, bibliometrics is widely used in research evaluation, academic tenure and promotion process, and grant application evaluation. Bibliometrics is also frequently used for journal ranking, university ranking, and even country ranking in terms of research output and standing.

Since its beginning in 1960s, when Eugene Garfield created the Science Citation Index (SCI), bibliometrics is considered a subfield of library and information science. Academic libraries around the world are particularly interested in the application of bibliometrics. There is a growing library involvement in bibliometrics by establishing dedicated library bibliometric services. In addition, academic libraries often collaborate with other campus units in bibliometrics data gathering and analysis for promotion and tenure process, grant application evaluation, hiring decisions, and program evaluation, etc.

There are generally three levels of metrics: journal-level, author-level, and article-level.

Journal-level metrics: journal impact factor or journal ranking score is used to rank the journals in the same discipline. The major products are Journal Citation Reports from Clarivate Analytics, CiteScore from Elsevier, and SCImago Journal and Country Rank (SJR). 
Author-level metrics: H-Index is one of the most widely used indicators to measure author's and researcher's productivity and impact. A researcher with a certain number of H-Index (h) means that researcher must have (h) publications, and each publication has received at least (h) citations. For example, an h-index of 15 means the researcher has at least 15 publications and each has been cited at least 15 times. H-Index can be retrieved in Scopus from Elsevier, Web of Science from Clarivate Analytics, and Google Scholar. In addition to H-Index, there are other author-level metrics, such as G-Index and i10-Index.

Article-level metrics: The major two metrics for article-level impact evaluation are the traditional citation counts, and the newer Internet-based alternative metrics or webometrics which measure the reach and influence of articles based on the online interactions such as online views, downloads, likes and shares on social media network and platform, blogs, etc.

In addition to the traditional and proprietary products in bibliometrics, there are now many new and some free tools and applications available either online or on desktop that requires download and local installation. The following is just an incomplete but very popular list: RBibliometrix package (desktop), ResGap.com (online), Publish or Perish (desktop), ImpactStory (online), VOSviewer (both online and desktop), Webometric Analyst (desktop), Unsilo Evaluate (online, an AI tool), citationgecko.com.

\section{MAJOR TRENDS OVER THE PAST DECADE}

Since 1960s, the traditional citation counting based bibliometrics has gradually become the standard practice in research evaluation and impact analysis throughout the scientific and academic world. In some places, metric and indicators have become the only criteria for tenure and promotion, and funding evaluation. The weakness and limitations of traditional bibliometrics are three folds.

First, any bibliometric tool or application such as Web of Science and Scopus has a limitation of scope in terms of the formats of materials inclusion, subject breadth, and subject depth. Second, due to the disciplinary differences, the impact of research output in humanities and social science is often not accurately represented. Last, there are some inherent limitations of any metric and indicator system. For example, co-authorship sometimes can produce very high citation count for certain researchers, but it does not reflect the true contribution and impact. Nowadays it is common to see one paper with dozens or hundreds of authors.

For the last twenty years, the research nature and output dissemination have gone through tremendous changes. There are more variety of output formats in addition to traditional journal articles and books. The Internet, especially social media networks, has gradually become an important venue in disseminating the research output and therefore their influence and impact should be taken account in evaluating research and researchers.

There are three major trends in the field of bibliometrics practice over the past decade.

The first trend is the advent and popularity of alternative metrics that supplements the traditional metrics. Two products stand out: Altmetrics and PlumX. Both try to gauge the influence and impact of research output by way of gathering and capturing the online user interactions, such as the count of online views, downloads, likes, sharing, mentions, etc. Alternative metrics or indicators have gradually become a standard part of scholarly communication to support research 
evaluation and academic tenure and promotion process. Many publishers have started to display publication's Altmetrics score and/or PlumX score of on their websites.

The second trend has to do with the responsible use of bibliometrics and responsive research evaluation movement. Responsible use of bibliometrics refers to the ethical and appropriate use of traditional citation-based metrics. Following are three major milestones.

Declaration on Research Assessment (DORA) (https://sfdora.org/): The Declaration on Research Assessment (DORA) recognizes the need to improve the ways in which the outputs of scholarly research are evaluated. The declaration was developed in 2012 during the Annual Meeting of the American Society for Cell Biology in San Francisco. It advocates the need to advance holistic and robust approaches by eliminating the practice of solely use of journal-based metrics, such as Journal Impact Factors, in funding, appointment, and promotion considerations.

Leiden Manifesto (http://www.leidenmanifesto.org/): In the comment published in April 22, 2015 issue of Nature, the authors proposed ten principles to guide research evaluation and to support the measurement of research performance, titled "Bibliometrics: The Leiden Manifesto for research metrics."

Hiroshima Statement from INORMS (https://inorms.net/): At the INORMS (International Network of Research Management Societies) International Congress in May 2021, the Hiroshima Statement was formally signed by members of the INORMS Council. The Statement contains five principles to promote best practices and guidelines in research evaluation and assessment.

Last but not the least, by way of the popularity of webometrics such as Altmetrics and PlumX, many universities and funding agencies requires that the candidates produce evidencebased narrative describing how their research has social impact.

The last trend is the use of AI and machine learning in bibliometrics. Using AI and full text mining technology, the new methods and tools in bibliometrics take advantage of the increasing availability of open access collections online to get fine-grained information about citation contexts. AI-based citation evaluation tools can classify citations into different categories, such as supporting, mentioning, disputing, and influential. Semantic Scholar and Scite.ai are two examples.

In conclusion, looking into the future, we are going to see more responsible use of bibliometrics in research evaluation and assessment, and application of AI and machine learning in bibliometrics.

\section{RESOURCES}

\section{Selected Publications}

1. Rousseau, Sandra; Rousseau, Ronald (2017). "Being metric-wise: Heterogeneity in bibliometric knowledge”. El profesional de la información, v. 26, n. 3, pp. 480-487. https://doi.org/10.3145/epi.2017.may.14

2. Ingrid Bauer, David Bohmert, Alexandra Czernecka, Thomas Eichenberger, Juan Garbajosa, Horia Iovu, ... Kurt De Wit. (2020, June 10). "Next Generation Metrics." Zenodo. http://doi.org/10.5281/zenodo.3874801 
3. Caroline S. Wagner, Loet Leydesdorff (2012). "An Integrated Impact Indicator: A new definition of 'Impact' with policy relevance". Research Evaluation, v. 21, n. 3, pp. 183188. https://doi.org/10.1093/reseval/rvs012

4. Parks, Sarah, Daniela Rodriguez-Rincon, Sarah Parkinson, and Catriona Manville (2019). "The changing research landscape and reflections on national research assessment in the future." Research England. https://www.rand.org/pubs/research_reports/RR3200.html

5. Wilsdon, J., et al. (2015). The Metric Tide: Report of the Independent Review of the Role of Metrics in Research Assessment and Management. https://doi.org/10.13140/RG.2.1.4929.1363

6. Wang, M., Wang, Z. \& Chen, G. (2019). "Which can better predict the future success of articles? Bibliometric indices or alternative metrics." Scientometrics, v.119, n.3, pp.1575-1595. https://doi.org/10.1007/s11192-019-03052-9

\section{Major Journals That Publish Either Exclusively or Heavily on Bibliometrics}

1. Scientometrics

2. Journal of the Association for Information Science \& Technology

3. DESIDOC Journal of Library Information Studies

4. Library Philosophy \& Practice

\section{Major Conferences}

1. LIS Bibliometrics Annual Event. https://thebibliomagician.wordpress.com/conferencesand-events/

2. International Conference on Scientometrics \& Informetrics. https://www.issisociety.org/conferences/

3. Altmetrics Conference (AM). https://www.altmetricsconference.com/

4. STI Indicators Conference Series. https://enid-europe.eu/index.php?id=confSeries

\section{Major Organizations and Associations}

1. LIS-Bibliometrics Community. Blog: https://thebibliomagician.wordpress.com/

2. Center for Science and Technology Studies (CWTS). https://www.cwts.nl/

3. International Society for Scientometrics and Informetrics (ISSI). https://www.issisociety.org/

4. European Network of Indicator Designers (ENID). https://enid-europe.eu/

5. International Network of Research Management Societies (INORMS). https://inorms.net/

6. Global Research Council (GRC). https://www.globalresearchcouncil.org/

\section{About the author}

Yongming Wang is the systems librarian at The College of New Jersey. He received his MLS from Rutgers University. His research interests include Library Service Platform, artificial intelligence and machine learning, data science and statistics, linked data, and research data management. He can be reached at wangyo@tcnj.edu. 